BNL- -46416

DE91 015844

\title{
Properties of a Symmetric RHIC Insertion
}

S. Y. Lee

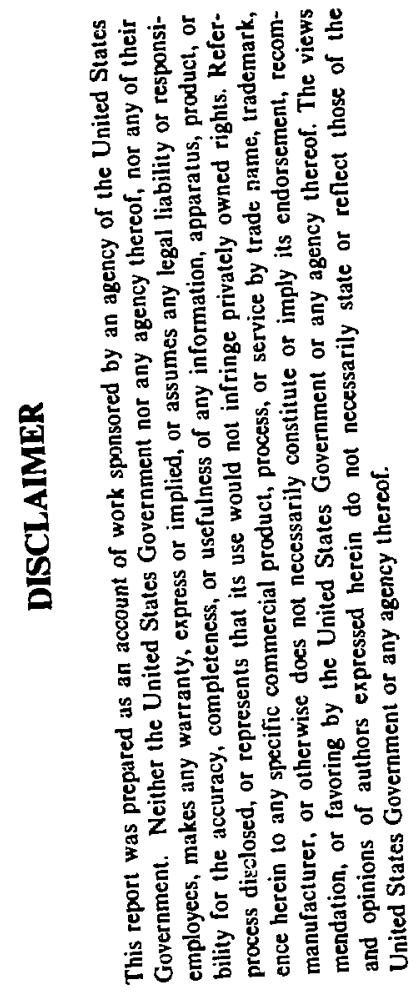

July 1991

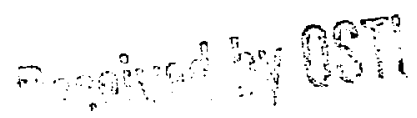

36131991

\section{R H I C P R O J E C T}

Broolhaven National Laboratory

Associated Universities, Inc.

Upton, NY 11973

Under Contract No. DE-AC02-76CH00016 with the UNITED STATES DEPARTMENT OF ENERGY 


\title{
Properties of a Symmetric RHIC Insertion ${ }^{\dagger}$
}

\author{
S.Y. Lee* \\ Brookhaven National Laboratory
}

\begin{abstract}
This report evaluates the lattice functions of the symmetric insertion proposed by $A$. G. Ruggiero for the RHIC insertion. the crossing geometry, Inner and Outer matching sections, and chromatic properties are studied in details. Some properties of the missing dipole dispersion correction scheme are also discussed. We found that the chromatic properties of the symmetric insertion is not better than the antisymmetric insertion. The problem is that the four family sextupole correction scheme seems not able to improve the chromatic distortion. Analytic understanding of the failure of the four family sextupole corrction scheme will be very useful.
\end{abstract}

† This work is performed under the auspieces of the U.S. Department of Energy.

- Permant Address: Department of Physics, Indiana University, Bloomington, IN 47405. 


\section{Introduction}

Recently, Ruggiero suggested a symmetric insertion for RHIC, where the quadrupoles of same polarity are placed symmetrically with respect to the crossing point. Fig. 1 shows a schematic layout of a RHIC insertion. There are 12 FODO cells in each arc. The insertion starts with a missing dipole dispersion suppressor consisting of two cells. The insertion matching section is composed of a FODO cell, a doublet and a triplet to achieve betatron function matching at the crossing point. The lattice function has a reflection symmetry at the interaction point (IP). Due to the intrinsic symmetry, the scheme is more attractive with a vertical layout. However, the hosizontal beam separation is relatively small in comparison with the total circumference, a horizontal layout will still retain reasonable symmetry. The study is based on the horizontal layout. Due to small symmetry breaking, the result of the present study shall remain valid for the vertical layout.

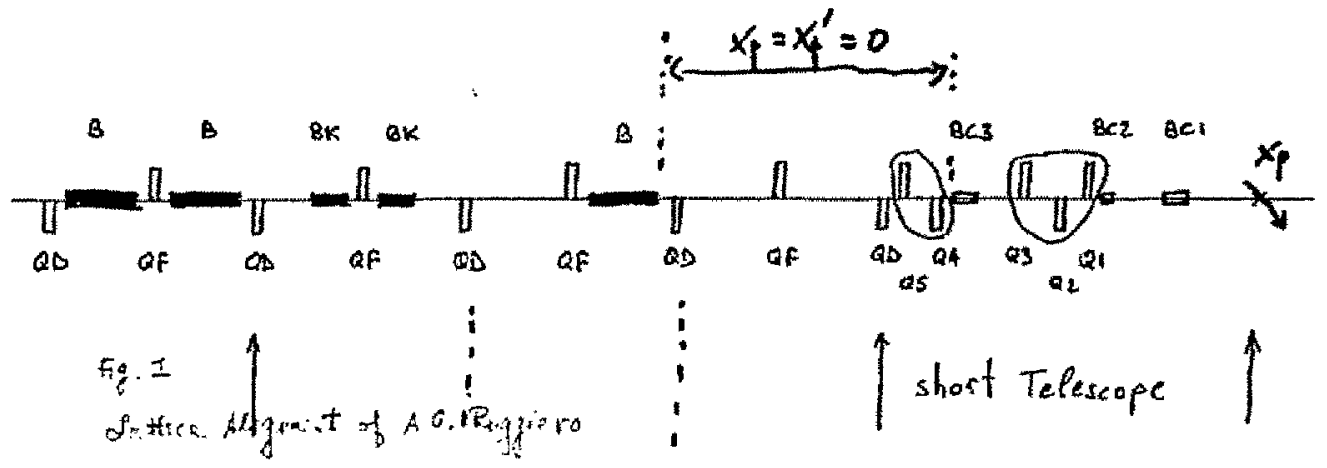

The paper shall address the physics of the beam dynamics issues of the symmetric insertion. In section 2, I discuss the required proper RHIC crossing geometry, the simplifcation of the matching section from that of Fig. 1. The chromatic properties of the lattice shall be studied as well. The conclusion will be given in section 3 . 


\section{Description of the Symmetric Insertion}

\subsection{The Crossing Geometry}

The crossing geometry for RHIC requires collision of equal and unequal species. To achieve the beam collision, two dipoles shown in the following configuration are needed. Let us call these two dipoles as BC1 and BC2 (see Fig.2).

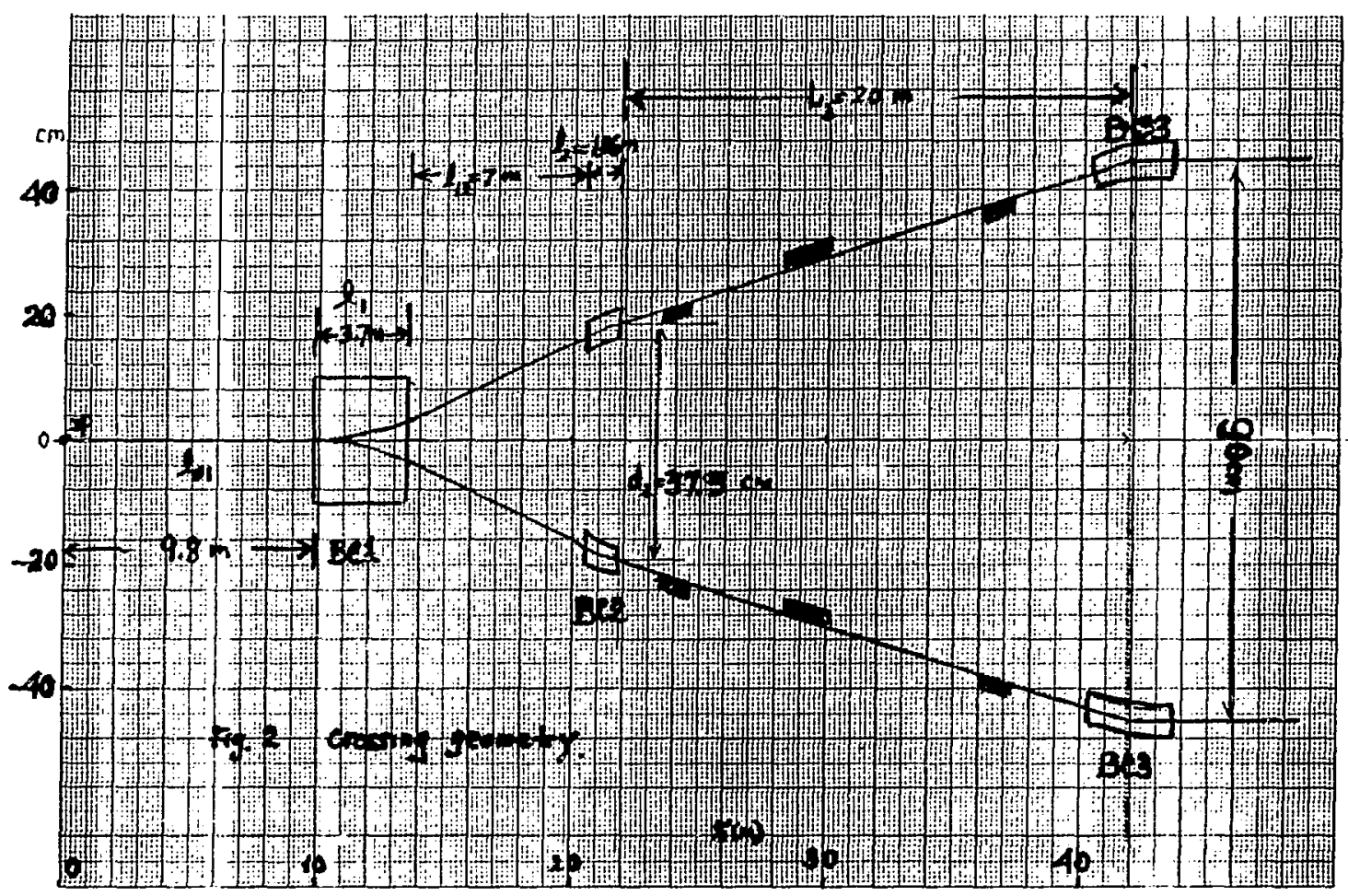

The field strength of these two dipoles has to obey the following basic equation.

$$
\ell_{01} \tan \alpha+\frac{\ell_{1}\left(\cos \alpha-\cos \theta_{12}\right)}{\sin \theta_{12}-\sin \alpha}+\ell_{12} \tan \theta_{12}+\frac{\ell_{2}\left(\cos \theta_{3}-\cos \theta_{12}\right)}{\sin \theta_{12}-\sin \theta_{3}}=d_{2}
$$

where

$\ell_{01}=$ distance between the interaction point and $\mathrm{BC} 1$

$\ell_{1}=$ length of $\mathrm{BC} 1$

$\ell_{12}=$ centerline distance between the $\mathrm{BC} 1$ and $\mathrm{BC2}$

$\ell_{2}=$ centerline length of $\mathrm{BC2}$

$\alpha=$ the angle between the beam and the centerline line 
$\theta_{12}=$ the angle of the beam and the centerline at the location between $\mathrm{BC} 1$ and $\mathrm{BC} 2$

$\theta_{3}=$ the angle of the beam and the centerline at the far end of the BC2

$d_{2}=$ the distance between two beam at the far end of the BC2

Note here that Eq.(1) should be solved for $\theta_{12}$ with given $\alpha$ and geometry constraints $\ell_{01}, \ell_{1}, \ell_{12}, \ell_{2}$ and $d_{2}$. The crossing angle between two beams of equal species is then $2 \alpha$. The Newton-Raphson's method can be used easily to solve Eq.(1). A program called XPARA.FOR to solve Eq.(1) is resides in th RHIC database in BNLDAG. Once $\theta_{12}$ is obtained, the bending angle of $\mathrm{BC} 1$ is given by $\theta_{1}=\theta_{12}-\alpha$. The radius of curvature for the $\mathrm{BC} 1$ is given by $\rho_{1}=\ell_{1} /\left(\sin \theta_{12}-\sin \alpha\right)$. The path length of the particle is then given by $s_{1}=\rho_{1} \theta_{1}$. The particle path length between $\mathrm{BC} 1$ and $\mathrm{BC} 2$ is given by $s_{12}=\ell_{12} / \cos \theta_{12}$. Similarly, the path length and the bending radius of $\mathrm{BC} 2$ can be obtained. Such a procedure is implanted in the RHIC lattice RHIC91. The procedure is also used in the symmetric lattice study.

In the following study, we shall used the geometry as defined in Fig.2, where a BC3 is used to bend two beams from $90 \mathrm{~cm}$ separation to $37.5 \mathrm{~cm}$ separation. We arbitrarily choose the distance $\ell_{23}$ between the far end of $\mathrm{BC} 2$ and the centribend point of $\mathrm{BC} 3$ to be 20 meters, which can accomodate the triplet focusing quadrupole elements. Note that when the distance $\ell_{23}$ is short, say $20 \mathrm{~m}$, the required dipole length of $\mathrm{BC2}$ will also be short. However the price one pays is that the $\mathrm{BC} 3$ aperture requirement will be the same as that of the large aperture quadrupoles. On the other hand, when $\ell_{23}$ is long, the length of $\mathrm{BC} 2$ becomes longer. The trade-off can be discussed in the technical point of view without much impact on the beam dynamics. The lattice property shall be independent of the loaction of $\mathrm{BC3}$, as long as there is space available. Therefore we shall choose the present configuration shown on Fig.2 for the study.

\subsection{Dispersion Control in the crossing region}

To obtain zero dispersion function, $D=D^{\prime}=0$, outside the crossing geometry region, and $D^{*}=0$ at the interaction point(IP), one needs quadrupoles between BC2 and BC3 dipoles. In the appendix, a minimum configuration of first order achromat is discussed, where the symmetric configuration can give dispersion correction outside the BC3. 
In the present configuration, a quadrupole-triplet can be used to provide dispersion correction as well as betatron function matching. We shall simplify the configuration of Fig. 1 to a doublet and a triplet configuration as shown on Fig. 3. The simplified configuration shall give identical flexibility of the originai lattice configuration (see Fig. 1). The quadrupole triplet, Q1, Q2, and Q3 and the quadrupole doublet Q4 and Q5 form the beam matching section, which adjoins the dispersion suppressor from the arc.
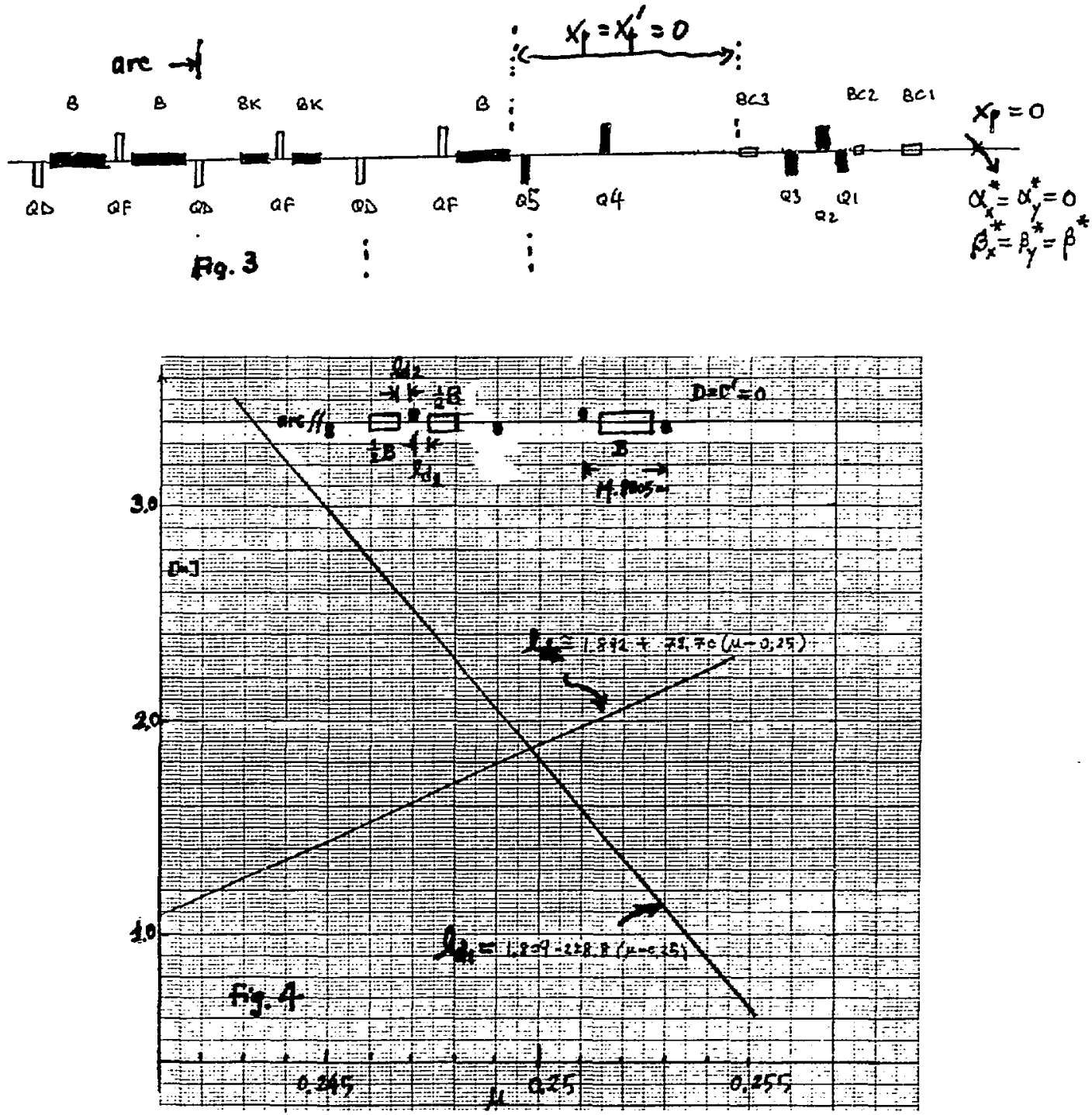
The dispersion suppressor is composed of missing dipole cells. Fig.4 shows the dispersion suppressor configuration, where the distances between the half dipoles and the adjacent quadrupoles are adjusted according to the phase advance of the FODO cell in the arc. Similar to RHIC91, there are 12 FODO cells in each arc. Each FODO cell has about $90^{\circ}$ phase advance. These distances depend weakly on the vertical betatron phase advance per cell in the arc. They are given by

$$
\ell_{d 1} \approx 1.809-228.8(\mu-0.25) ; \quad \ell_{d 2} \approx 1.892+78.70(\mu-0.25)
$$

Alternately, one can also adjust the gradient in achieving the dispersion correction. The phase advance between the quadrupoles will be different from $90^{\circ}$. Using the space adjustment, the operational window for the phase advance in the arc cell is $0.24 \leq \frac{\mu}{2 \pi} \leq \mathbf{0 . 2 5 4}$. This requirement arises from a minimum distance of $1 \mathrm{~m}$ between quadrupole and dipole.

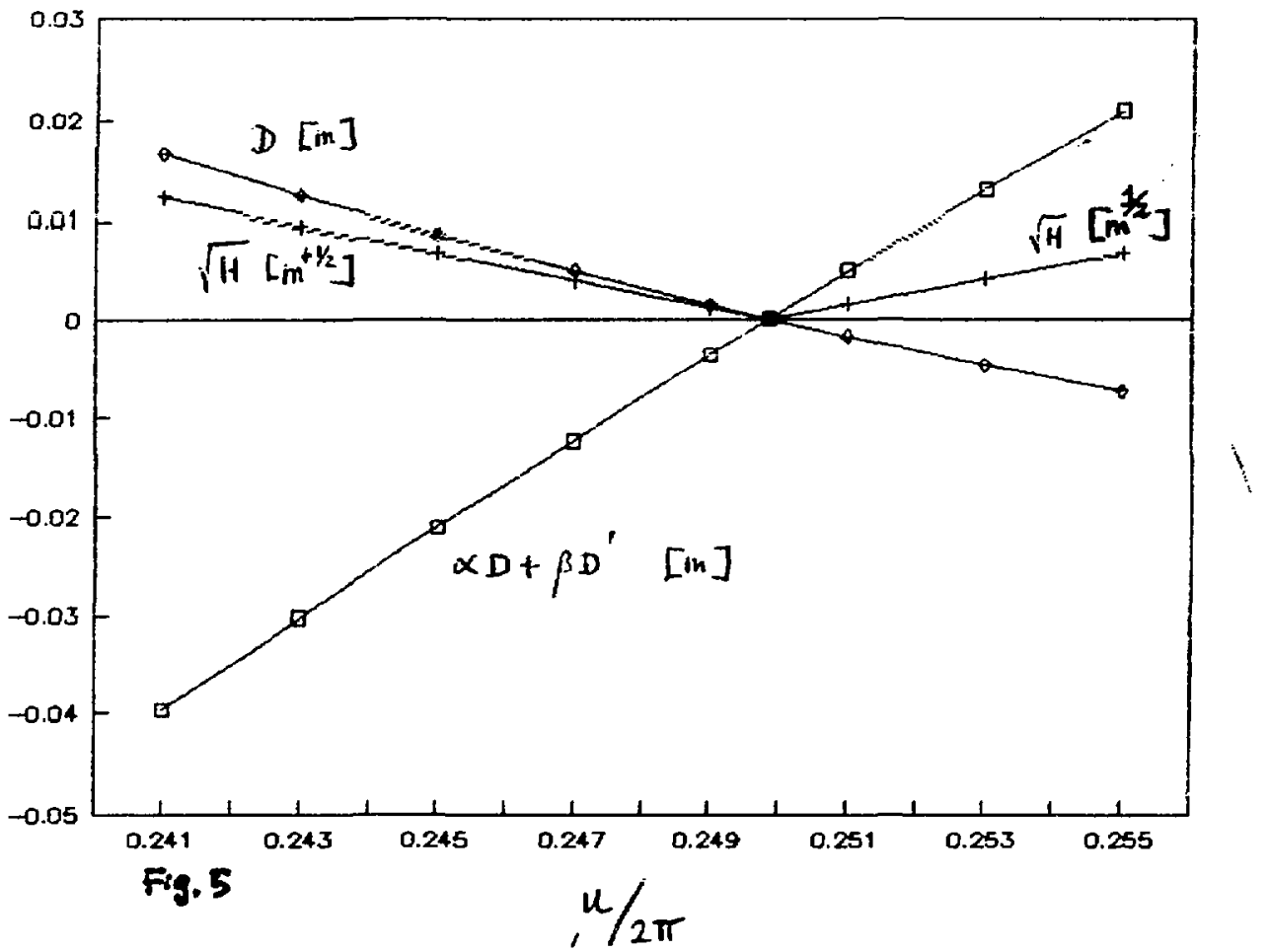

Once the distances $\ell_{d 1}, \ell_{d 2}$ are determined, the change of the machine betatron tunes can be achieved by changing the phase advance per cell $\mu_{x}, \mu_{y}$. Thus the dispersion suppressor will not be perfect. The effect of mismatch in the dispersion function is given by 
Fig. 5 , where the $D_{x}, \gamma_{x} D_{x}+\beta_{x} D_{x}^{\prime}$, and the $\sqrt{H}$ with

$$
H=\frac{1}{\beta_{x}}\left\{D_{x}^{2}+\left(\gamma_{x} D_{x}+\beta_{x} D_{x}^{\prime}\right)^{2}\right\}
$$

are ploted as a function of the betatron phase advance per cell. Note that the invariant function $H$ remains small. The dispersion function in the mismatch situation is given by $\sqrt{\beta_{x} H}$.

2.3 The optimization of $\beta^{*}=6 \mathrm{~m}$ lattice

The strength of quadrupoles Q1-Q5 and the spacing between these quadrupoles are optimized to obtain a proper betatron amplitude functions at the crossing point (or the interaction point IP).

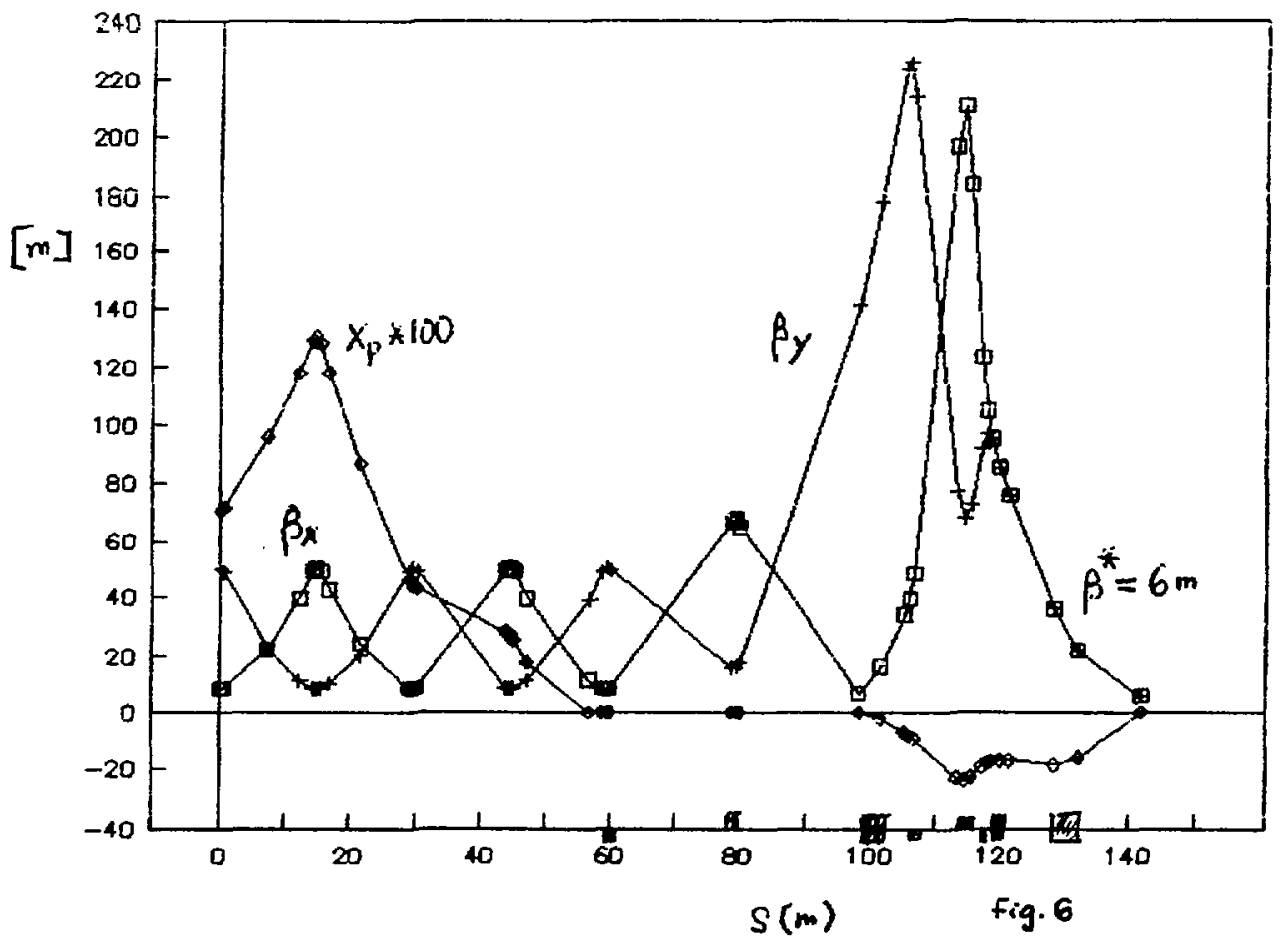

A possible solution is shown in Fig.6, where the betatron amplitude functions are optimized to $\beta_{x}^{*}=\beta_{y}^{*}=6 \mathrm{~m}$. Fig. 6 shows a half insertion. The full insertion is composed of a nearly mirror symmetry with respect to the interaction point (IP). By properly using 
the inner and outer arcs and inner and outer insertions, the RHIC lattice with $\beta^{*}=6 \mathrm{~m}$ can be studied. The tunes of the machine is at $Q_{x}=33.7710$ and $Q_{y}=29.6132$ with natural chromaticity $\xi_{x}=-58$ and $\xi_{y}=-48$. Since the study of the bare ideal lattice is insensitive to the betatron tune.

\section{3a Betatron Tuning of the RHIC symmetric Lattice}

A. Minimum Constraint Tuning

The minimum constraint tuning requires the following symmetric conditions and the $\beta^{*}$ condition:

$$
D^{*}=\alpha_{x}^{*}=\alpha_{y}^{*}=0 ; \quad \beta_{x}^{*}=\beta_{y}^{*}=\beta^{*} .
$$

There are five constraints needed in the $\beta^{*}$ adjustment at the IP. Therefore five parameters are needed. The phase advance will change during the the $\beta^{*}$ tuning procedure. Table 1 shows the fitting procedure without cnstraint on the maximum betatron amplitude function.

Table 1. The maximum betatron amplitude functions vs $\beta^{*}$

\begin{tabular}{|c|c|c|c|c|c|c|c|c|c|c|c|c|c|}
\hline$\beta^{*}(\mathrm{~m})$ & 2 & 2.5 & 3 & 3.5 & 4 & 4.5 & 5 & 5.5 & 6 & 6.5 & 7 & 7.5 & 8 \\
\hline$\beta_{x}^{\max }(\mathrm{m})$ & 492 & 389 & 341 & 305 & 277 & 255 & 237 & 222 & 211 & 198 & 183 & 169 & 158 \\
$\beta_{y}^{\max }(\mathrm{m})$ & 1289 & 983 & 744 & 584 & 470 & 395 & 323 & 271 & 226 & 205 & 203 & 201 & 200 \\
\hline
\end{tabular}

It is clear that the maximum betatron amplitude function in this fitting procedure is not practical. The dynamical aperture will be too small to be acceptable. The final maximum betatron function at $\mathrm{Q} 3$ location will be too large.

B. Maximum betatron function constraint

Alternately, one can try to control $\beta_{y}^{\max }$ by requesting an extra condition:

$$
\beta_{y}^{\max } \leq \frac{1350}{\beta_{y}^{*}} .
$$

where the number 1350 in Eq.(3) is derived from the constraint on the quadrupole gradient and the minimum distances between the interaction point and the quadrupole locations. The resulting maximum betatron function is more respectable. However, such a constraint will contradict the requirement of $D^{*}=0$. 
Unfortunately, there are more constraints than adjustable parameters. The resulting $D^{*}$ is a nonzero small number of a few millimeters, which however create mismatch of dispersion function at Q2 of the order of $\pm 0.8 \mathrm{~m}$ at $\beta^{*}=2 \mathrm{~m}$ instead of the matched $\pm 0.25 \mathrm{~m}$. Fig. 7 shows the resulting betatron amplitude function and the contribution of natural chromaticity per insertion. Note that in such a fitting procedure, the quadrupole strength for Q1-Q3 are nearly constant.

Fig. 8a shows the integrated quadrupole strength requirements in the betatron tuning. During the $\beta^{*}$ tuning, the phase advance across the insertion is also changing. Fig. $8 \mathrm{~b}$ shows the change of the phase advance in the above tuning procedure. To compensate the phase advance change in the insertion, the phase advance per cell should also be changed accordingly to maintain a constant betatron tunes.

2.3b Chromatic properties of the symmetric insertion lattice

Due to the ransition energy crossing and the intrabeam Coulomb scattering, the resulting momentum spread is $\mathbf{\pm 0 . 0 0 5}$. The RHIC lattice requires excellent chromatic properties. The variation of the betatron tunes and the betatron amplitude functions are shown in

Fig 9 for $\beta^{*}=2 \mathrm{~m}$ with two family sextupole correction scheme. It worth point out that four family sextupole scheme does not help to reduce the chromatic distortion.

\subsection{Proper Machine Tuning}

It is known that the dynamical aperture becomes more important during the minibeta squeeze. Therefore, the betatron functions should be properly matched for the low beta insertion. When the machine is tuned for the higher-beta value for the injection, the accelerator should be more tolerable because of smaller betatron amplitude function at the high beta triplet.

To avoid the structure resonances in an accelerator with six fold symmetry, we have to choose the betatron tunes away from integers, such as $27,30,33,36$. For the symmetric insertion with $90^{\circ}$ phase advance per cell in the arc(Section 2.2), the tunes occur naturally at $Q_{x}=34.827$ and $Q_{y}=28.723$. It is possible to change the betatron tunes by 1 from the previous discussions of the dispersion suppressor.

Indeed the symmetric insertion in the RHIC lattice posses systematic half integral 

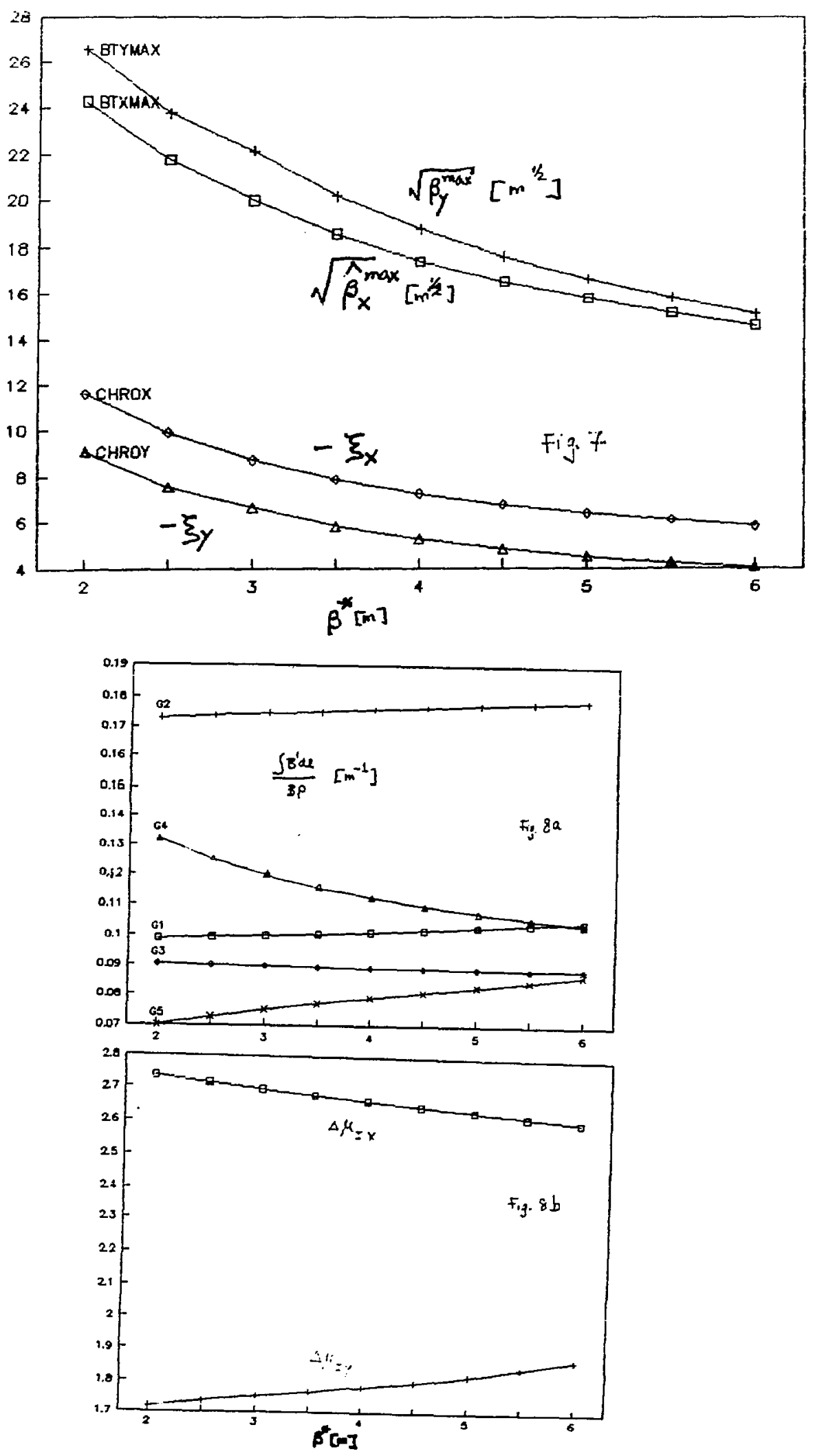

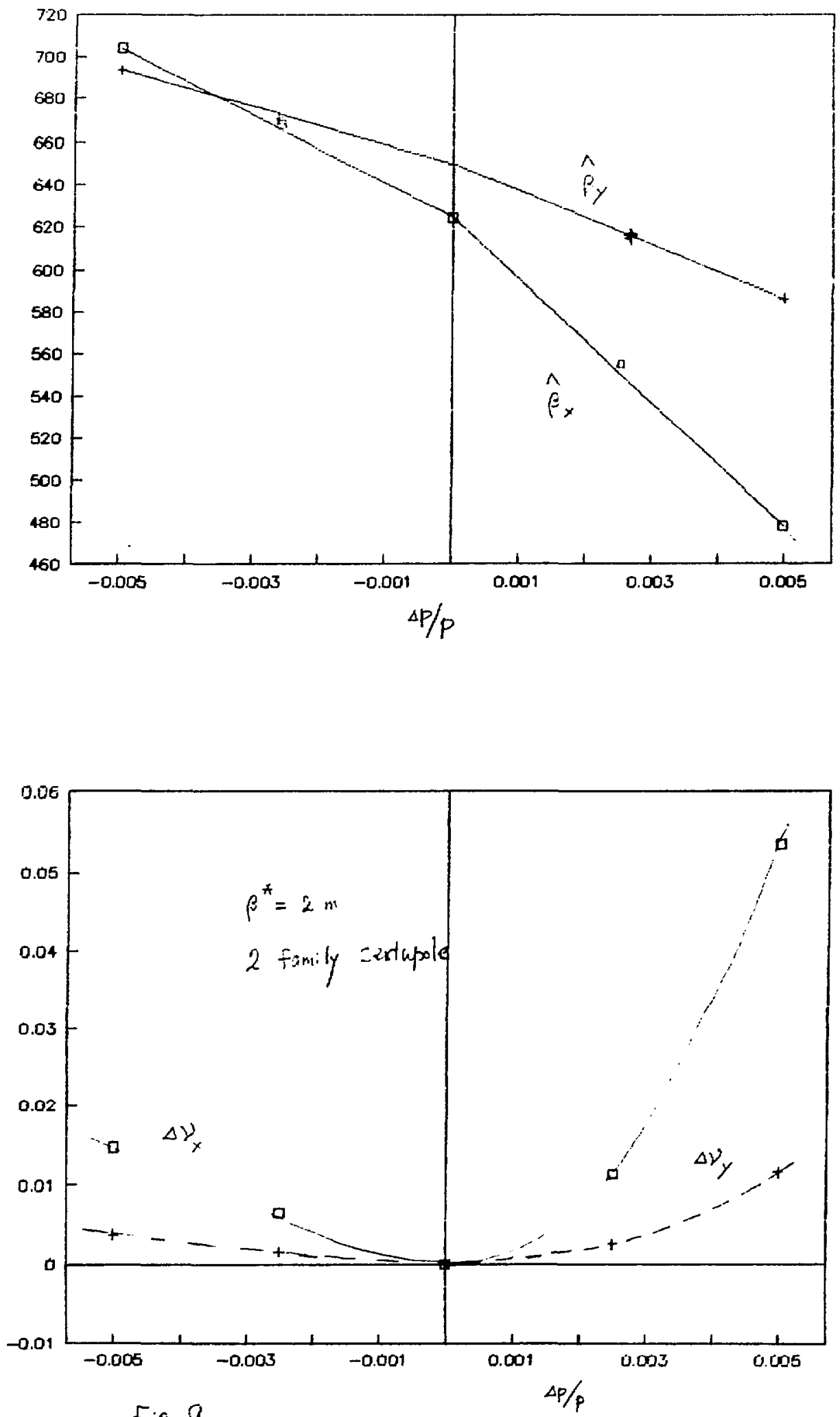

Fig. 9 
stopbands at tune values of $27,30,33,36$ etc.. Our tunes are sufficiently far away from these stopbands. The horizontal and vertical betatron tunes are therefore adjusted to $Q_{x}=34.827$ and $Q_{y}=28.823$. The SYNCH input and output files for the symmetric insertions are available in the BNL cluster vax: \$2\$DUA:[RHIC.LEE]RHICSYM.OUT;2 RHICSYM.OUT;3 RHICSYM.DAT;2 RHICSYM.DAT;3. Another file RHICSYM.DAT;4 RHICSYM.OUT; 4 are the lattice properties for $Q_{x}=33.826$ and $Q_{y}=28.823$. These files for the $\beta^{*}=2 \mathrm{~m}$ show however large chromatic variation, which is hard to be corrected by four family sextupole scheme. The Q2 quadrupole gradient requirement for the file versions 2 is about 69 Tesla/meter instead of the norminal 57 Tesla/meter. Similarly, the $\beta^{*}$ tuning are performed in the file \$2\$DUA7:[RHIC.LEE]RHICSYMTB.OUT.

\section{Conclusions and Discussions}

We have studied the beam dynamics properties, tunability, chromatic properties of the symmetric insertion for RHIC. We found that the combination of the triplet Q1, Q2 and Q3 for the dispersion function matching and the optical matching gives larger nagative natural chromaticity than the corresponding antisymmetric lattice. Five adjustable parameters are not enough degree of freedom to obtain excellent matching, yet the mismatch is indeed small. The only problem is that two family sextupole correction scheme can not achieve good chromatic correction, yet four-family scheme does not work at all. The reason that four family does not work needs to be understood. It is an interesting problem of its own right. The lattice tuning has been demonstrated in the files resides in the RHIC directory, \$2\$DUA7:[RHIC.LEE]. Further works are needed if the lattice would be chosen as the RHIC lattice. The magnet requirement in the symmetric lattice is the same as that of the antisymmetric lattice except the BC3 dipole which should be a $130 \mathrm{~mm}$ coil i.d. similar to that of large aperture quadrupoles.

\section{Appendix}

A. First order achromat for the beam crossing

To achieve a first order achromat in the beam crossing geometry with dipoles B1 and B3, which deflect beams onto collision course, it is usually desirable to demand dispersion functions $D_{x}=D_{x}^{\prime}=0$ outside the crossing region and $D_{x}=0$ at the interaction point. 
The achromatic condition requires a focusing quadrupole to be located between B1 and B3. Using a thin lense approximation for the dipole and quadrupoles, then the focal length, $f$, of the quadrupole is given by

$$
\frac{1}{f}=\frac{\ell_{0}+\ell_{2}+\ell_{3}}{\ell_{3}\left(\ell_{0}+\ell_{2}\right)}+\frac{\phi_{3}}{\phi_{1}} \frac{\ell_{0}}{\ell_{3}\left(\ell_{0}+\ell_{2}\right)}
$$

where

$$
\begin{aligned}
& \ell_{0}=\text { the distance between the IP and the thin dipole B1 } \\
& \ell_{2}=\text { the distance between the thin dipole B1 and the thin quad } \\
& \ell_{3}=\text { the distance between the thin quad and the thin dipole B3 } \\
& \phi_{1}=\text { the bending angle of the thin dipole B1 } \\
& \phi_{3}=\text { the bending angle of the thin dipole B3 }
\end{aligned}
$$

Since $-1 \leq \frac{\phi_{8}}{\phi_{1}}$, a focusing quadrupole is needed in the achromatic condition. For a parallel beam line with head on collision, we have $\phi_{3}=-\phi_{1}$. The corresponding focal length becomes,

$$
\frac{1}{f}=\frac{\ell_{2}+\ell_{3}}{\ell_{3}\left(\ell_{0}+\ell_{2}\right)}
$$

The scheme requires symmetric insertion, i.e. the quadrupoles are of the same polarity on both side of the interaction point. 